\title{
Information Communication Technologies as Tools to Cope with Stigma: An Exploration of Coping in College-Aged Queer People of Color
}

\author{
Beatrice H. Fadrigon, B.S. ${ }^{1,5}$, Courtney E. Smith ${ }^{2,5}$, Chantelle A. Roulston, B.A.* ${ }^{3,5}$, \\ Juan F. Maestre ${ }^{4}$ \\ ${ }^{1}$ University of Pittsburgh, Pittsburgh PA 15213, USA \\ ${ }^{2}$ Pennsylvania State University, University Park 16802 USA \\ ${ }^{3}$ Stony Brook University, Stony Brook NY 11790 USA \\ ${ }^{4}$ Indiana University, Bloomington IN 47405 USA \\ ${ }^{5}$ The iSchool Inclusion Institute, Pittsburgh PA 15213 USA \\ *Corresponding Author: Chantelle A. Roulston, B.A., chantelle.roulston@stonybrook.edu
}

\begin{abstract}
Within the United States, Queer People of Color (QPoC) experience high levels of societal discrimination and oppression as a result of having both a stigmatized racial identity and a stigmatized sexual orientation/gender identity. Despite this, QPoC have been the focus of very few studies, and little is known about how to effectively support this marginalized group. Research shows that QPoC utilize Information Communication Technologies (ICTs) to cope with societal stigma, however few studies have addressed exactly what ICTs QPoC are using and how they are using them. This qualitative study explores common themes in the experiences of stigma for QPoC, what ICTs $\mathrm{QPoC}$ are using to cope with this stigma, and how they are using these ICTs. The authors conducted 12 semi-structured interviews followed by a thematic analysis. The main ICTs that participants discussed using include: Instagram, Twitter, Discord, Tinder, Grindr, GroupMe, Tumblr, Reddit, Netflix, YouTube, Video Games, and Texting. These tools were primarily used for distracting and escaping from stigma, communicating and connecting with others, seeking QPoC media, exploring one's identity, seeking a community, and finding emotional support. Participants reported that these ICTs are effective coping mechanisms, however stigma permeates these online spaces as well, making it difficult for QPoC to feel safe from the stigma they face offline. To address this, the authors put forth several suggestions.
\end{abstract}

Keywords: ICTs/Information Communication Technologies, Queer, Stigma, POC/People of Color, LGBTQ, HCI (Human Computer Interaction) 


\section{$1 \quad$ Introduction}

Marginalized groups such as people of color ( $\mathrm{PoC}$ ) and sexual/gender minorities including Lesbian, Gay, Bisexual, Transgender, and Queer individuals ("LGBTQ+" or "Queer" community ${ }^{2}$ experience high levels of stigma in the United States (U.S.) [12]. Stigma is defined as any attribute an individual has that differentiates and classifies them negatively within a particular identity category [1]. Many LGBTQ+ individuals in the U.S. have increased negative experiences throughout their lifetime as a result of societal stigma against the LGBTQ+ community [41, 8]. LGBTQ youth have a higher susceptibility of self-injury and depressive symptoms and are more likely to die by suicide [41]. Additionally, racial minorities experience higher rates of psychological distress and psychiatric symptoms related to stigma than individuals who do not identify as a racial minority $[1,3]$. As identities do not exist in isolation, it is possible to hold more than one stigmatized identity. Identifying as both a racial and sexual/gender minority creates an elevated risk of discrimination beyond already present discrimination that comes with having only one of these marginalized identities [40]. Queer people of color (QPoC) experience this elevated discrimination. Minority stress theory demonstrates that experiences of stigma, discrimination, and prejudice can create stressful social environments for marginalzied groups and can contribute to mental health problems [27]. Furthermore, intersectionality theory explains that individuals with more than one marginalized identity, such as $\mathrm{QPoC}$, may experience increased risk of stigma and discrimination, more so than individuals with more than one marginalized identity [11].

Many QPoC experience discrimination on a daily basis in the form of bullying, lower employment rates, and differential treatment in social settings, which can increase their risk of emotional distress and other mental health issues [12]. To ameliorate the negative emotional consequences of the stigma-driven discrimination $\mathrm{QPoC}$ are faced with, such as homophobia, transphobia, and racism, QPoC often develop resiliency and other forms of coping mechanisms [23], such as using the technology available to them. Literature suggests that there are two main uses of coping: focusing on problems or emotions to evade the stress from the current environment, or taking actions in the environment itself [24]. Coping is considered both a defense mechanism and an automatic response to deal with stressors, anxiety, and imminent danger; it is used flexibly in different environments depending on the situation and available resources [25]. Recent research on coping studies recommend that those who expect to be in stressful situations should prepare by having resources in mind to lessen the stress of that situation [17]. For college aged individuals (18-23) whose technology use is near ubiquitous in the United States, using technology may lessen the stresses of academia and of the experience of stigma. Pew Research Center reports about $95 \%$ of individuals who belong to this group own a smartphone and that

${ }^{1}$ LGBTQ+ stands for lesbian, gay, bisexual, transgender and queer (or questioning) and others [32]. Throughout the paper we use both the terms 'LGBTQ' and 'LGBTQ+' to reflect the language utilized in the referenced papers.

2 An umbrella term used to describe a sexual orientation, gender identity or gender expression that does not conform to dominant societal norms. While it is used as a neutral, or even a positive term among many LGBT people today, historically "queer" was used as a derogatory slur [19]. 
more than nine in ten undergraduates $(95 \%)$ are home high-speed internet users (this is above the national adult average of 66\%) [35]. Currently this age group, who is part of Gen Z (born 1997-2012), is the most "diverse generation in history" [18], and only about two-thirds of Gen Z identify as straight or heterosexual [36]. Given the sexual orientation demographics of this age group, it follows that many QPoC leverage Information Communication Technologies (ICTs) as a tool for coping [12, 41].

While extant literature suggests QPoC use ICTs in general for coping, little is known about which specific tools are being used and how. There is considerable research focusing on various ways $\mathrm{PoC}$ cope with stigma and the various ways LGBTQ+ individuals cope with stigma, however few studies focus on both simultaneously [31]. This research aims to explore how individuals with both of these identities experience stigma in their everyday lives, as well as how they leverage ICTs as a tool to facilitate coping. By centering the voices of these doubly marginalized individuals, a lot can be learned about the tools they use. This has the potential to prevent larger consequences of emotional distress and negative impacts on mental health. This study explores the research questions:

RQ1: How do QPoC college students, ages 18-23, in the US experience stigma?

RQ2: How do QPoC use information communication technologies (ICTS) to cope and specifically, what ICTs are they using?

\section{Background}

Stigma is defined as any attribute or something an individual has that classifies them negatively within a particular identity category [1]. People of color $(\mathrm{PoC})$ and sexual/gender minority individuals (e.g. lesbian, gay, bisexual, transgender, queer, and other [LGBTQ+ or Queer]) experience stigma against their identities in the United States [39]. Stigma can present itself in many forms including bullying, harassment, and discrimination [37]. Individuals who identify as both $\mathrm{PoC}$ and as Queer (QPoC) experience marginalization against both of their identities; they often experience high levels of stigma, societal discrimination, and oppression in the U.S., higher than individuals with only one of these two identities $[3,5]$. This has negative emotional consequences for $\mathrm{QPoC}$ and is linked to adverse mental health effects including depression, anxiety and somatization $[7,3]$.

The consequences of stigma can be pervasive and can often affect aspects of PoC's everyday life including their health, income, and perception by society $[30,23$, 31]. Manifestations of negative racial attitudes (e.g., microaggressions) can be psychologically distressing for PoC [19]. Similarly, LGBTQ+ individuals experience the effects of stigma, including cyberbullying at relatively high rates and workplace mistreatment stemming from one's sexual orientation $[18,3]$. Intersectionality theory posits that having multiple non-dominant identities may be connected with increased risk for negative experiences and health outcomes due to compounded effects of 
oppression associated with each individual identity [11]. A recent review of the literature regarding the impact of stigma on $\mathrm{QPoC}$ found that there is a clear distinction between having a singular marginalized identity and having more than one [36]. Many QPoC experience unique stressors related to their multiply-marginalized identities; this includes, for some, difficulty finding complete safety within either of their identity groups due to racial stigma within the LGBTQ community and sexual/gender stigma within PoC communities [34].

Minority stress theory explains QPoC tend to build resilience to societal stigma-based stress [5]. Existing literature has supported the fact that multiply-marginalized individuals often adopt coping mechanisms in response to stigma, for example reaching out to individuals who identify similarly though online communities [7, 18]. However, research regarding how QPoC in particular cope with the experience of stigma is extant. In fact, a recent literature review conducted by Roulston et al. found that there is considerable research that centers coping strategies for PoC and Queer individuals (e.g. social support is a proactive coping strategy when encountering stigma against race or sexual orientation [9]), however there is little work that centers QPoC coping specifically [36]. Current research suggests that many QPoC use information communication technologies (ICTs) as coping tools in response to stress brought on by societal stigma [12], however apart from the use of online communities, there is a lack of detailed knowledge on the potentially many ways QPoC are employing ICTs as a coping tool [36].

Previous research has centered minority individuals and underrepresented groups in Human-Computer Interaction (HCI) through focusing on feminist and queer theories [4, 6]. For example, Bardzell's work in 2010 provided a space for the examination of how technologies construct and perpetuate gender and offered opportunities to draw on feminism more in HCI research in a way that encompasses both theory and design practice [4]. Compared to research on socio-economic class and gender there is less research on ethnicity and race [33]. Within the field of HCI, researchers have recently had a hard time breaking down the complexity of what a "user" on the internet is related to the attributes and intersectionality of an individual's identity [33]. In a criticism of $\mathrm{HCI}$ as a research community, authors Ogbonnaya-Ogburu et. al wrote about ways to incorporate Critical Race Theory into the HCI community. This theoretical framework was first introduced in the 1970s as a way to challenge the dominant discourse on race and has now been incorporated into HCI research. Critical Race Theory puts forth several key tenets that are central to this paper: racism is ordinary, identity is intersectional, and there is a uniqueness to the voice of color [29]. This paper extends the existing body of work centering underrepresented populations through focusing on the unique experiences of a multiply-marginalized group who has been overlooked in HCI: QPoC.

More than 130 million individuals in the U.S. identify as both a PoC and as LGBTQ+ [35]. Many of these individuals may feel the need to develop coping 
mechanisms in response to racial and sexual/gender stigma at some point in their lives, and in the 21 st century it is likely that ICTs will play a role in this. With a better understanding of how QPoC leverage ICTs as a coping tool, it will be possible to maximize the positive effects of coping and minimize the negative effects of stigma.

\section{$3 \quad$ Methods}

\subsection{Data Collection}

The authors conducted a qualitative study involving 12 semi-structured interviews, at which point they reached saturation of information [20]. Each author conducted four to five interviews. For this study, each author received Institutional Review Board approval at their respective universities. Participants were recruited from October to December in 2019 using the following methods: physical and electronic flyers posted high areas of traffic (common areas on campus and instagram stories), social media, group chats, emails, and visiting queer communities. Participant eligibility criteria included being 18-23 years old and enrolled in a college or university; identifying as a person of color and as a part of the LGBTQ+ community or as queer.

The authors developed a series of open-ended interview questions to capture a well rounded and clear understanding of the experience of stigma for college aged QPoC as well as their use of technology-based coping mechanisms in response to this stigma.

Interviews began with open-ended questions regarding participants' self-reported identities. Participants then shared their feelings about their stigmatized identities, positive experiences, and experiences of discrimination or prejudice, stereotypes, racism, and homophobia. Participants were later asked about coping methods they employ, their effectiveness, and frequency of use to get a broad understanding of QPoC coping strategies in general. This introduced coping with stigma before delving into questions about technology used in this context. These questions covered types of technology they use generally, technology-based coping mechanisms, perceived helpfulness/effectiveness of online coping, and suggestions for future improvement of technology. Interviews lasted approximately 40 minutes and were securely audio recorded.

When the interviews were complete, each researcher individually transcribed and de-identified the interviews they conducted to maintain confidentiality before sharing the transcripts with others on the team. Interview transcripts were uploaded to a private, shared drive and contained no personal identifiers of the participants.

\subsection{Ethical Considerations}

Each author partnered with various PoC/LGBTQ+/QPoC organizations on their respective campuses with whom they had pre-existing relationships. All participants volunteered to be part of the study and could drop out at any time with no consequences. Most of the authors of this paper hold a personal stake in this research, as they identify as QPoC themselves. All authors abided by ethical guidelines 
proposed for this research with stigmatized populations. All procedures, recruitment material, and protocols received IRB approval at each authors' affiliated university.

Table 1. Participant Demographics

\begin{tabular}{|c|c|c|c|c|c|}
\hline $\begin{array}{l}\text { Participant } \\
\text { Code }\end{array}$ & Age & Race/Ethnicity & $\begin{array}{l}\text { Gender } \\
\text { Identity }\end{array}$ & $\begin{array}{l}\text { Sexual } \\
\text { Orientation }\end{array}$ & Pronouns \\
\hline $\mathrm{P} 1$ & 19 & Black & $\begin{array}{l}\text { Non- } \\
\text { binary }\end{array}$ & Bisexual & $\begin{array}{l}\text { They/Them } \\
\text { and She/Hers }\end{array}$ \\
\hline $\mathrm{P} 2$ & 20 & Black & Woman & Pansexual & She/Her \\
\hline P3 & 20 & Asian (Chinese) & $\begin{array}{l}\text { Cis-Wo } \\
\text { man }\end{array}$ & Lesbian & She/Hers \\
\hline P4 & 19 & Black & $\begin{array}{l}\text { Non- } \\
\text { binary }\end{array}$ & Lesbian & $\begin{array}{l}\text { She/Her and } \\
\text { They/Them }\end{array}$ \\
\hline P5 & 20 & $\begin{array}{l}\text { Half Black, Half } \\
\text { Hispanic }\end{array}$ & Man & Homosexual & $\mathrm{He} / \mathrm{Him}$ \\
\hline P6 & 22 & Non-white Latinx & Cis Man & Gay & $\mathrm{He} / \mathrm{Him}$ \\
\hline P7 & 20 & Asian & Woman & Bisexual & She/Her \\
\hline P8 & 18 & African-American & Woman & Bisexual & She/Her \\
\hline P9 & 22 & Black American & Man & Gay & $\mathrm{He} / \mathrm{Him} / \mathrm{His}$ \\
\hline P10 & 21 & Black & Man & Gay & $\mathrm{He} / \mathrm{Him} / \mathrm{His}$ \\
\hline P11 & 20 & Black & Woman & Asexual & She/Her/Hers \\
\hline P12 & 21 & $\begin{array}{l}\text { Afro-Caribbean } \\
\text { (Black) }\end{array}$ & $\begin{array}{l}\text { Gender } \\
\text { Fluid }\end{array}$ & Bisexual & They/Them \\
\hline
\end{tabular}

\subsection{Data Analysis}

Interview transcripts were analyzed with a thematic analysis approach [5]. This method involves identifying patterns of meaning in the data. The interview transcripts were uploaded to the secure, open source, qualitative analysis coding software Taguette. Taguette allowed the team to code the data by creating various tags for analysis. Authors agreed upon the criteria for each tag, for example all mentions of social media were assigned the "technology - social media" tag. Each transcript was 
coded independently prior to group meetings; disagreements were resolved through discussion. Authors coded each interview that they conducted themselves. Additionally, each author reviewed one another's codes for reliability and consistency, thus all twelve interviews were reviewed by all four authors. All authors met weekly for eight weeks to discuss and update the codes; final codes were applied across the data. Authors summarized and discussed the main ideas drawn from these codes, and through this analysis several themes emerged. The authors created a list of the most salient, recurring themes evident in the data and grouped them into categories from most to least frequent based on the number of excerpts assigned to each code.

\section{$4 \quad$ Findings}

\subsection{RQ1: Stigma Experiences}

As participants recounted their experiences with stigma, it became clear that the experience of stigma in online and offline settings are distinct. This section of the findings is separated into offline experiences followed by online experiences to reflect this.

Offline Experiences. Stereotyping is a common experience for QPoC. Stereotypes are not often hidden, rather are publicly made clear. One participant who identified as Black said that there is a clear expectation for Black people to be loud, violent, and untrustworthy. Another participant experienced a teacher yelling at their predominantly Black class, saying, "this is not the hood" (P4), despite the fact that most students in the class lived in the suburbs their whole lives. Another participant shared that he tries to hide his sexual orientation when he is in a predominantly Black setting because he knows he would be openly stereotyped.

Overt discrimination is also common experience for QPoC. Many participants indicated that the stigma associated with race is often stronger than the stigma associated with sexual orientation, likely because race is not a concealable identity whereas sexual orientation often is []. Exemplifying this point, P7 shares, "The people of color part of my identity is harder in the social or work area than the gay one." Multiple participants shared they had been called the $\mathrm{N}$ word before and recalled living with a sense that they do not fit in nor belong. One participant explained: "there are places Black people can't go without thinking of serious dangers for themselves because of the way they look" (P4). Participants also described bullying in high school, including homophobic comments, name calling, being spat on, and being called slurs in public. One person was even turned down for a babysitting job because the family "didn't want to make their kids gay" (P6), and another experienced a stranger saying they would pray for her after she kissed her partner on a public bus.

Many QPoC feel they have to hide parts of themselves and behave differently because of stigma. One participant described feeling pressure to be hyper-feminine and feeling invalidated if they did not. "I have to be hyper-feminine... if I don't express myself in a certain way then I'm not valid" (P1). Another participant believes they have to present as masculine, be a provider, and carry pains and trauma to fit the 
stereotype of a Black male. Sometimes this pressure even comes from participants' families. One participant explained that her mother is her best friend, but she is more scared to come out as bisexual to her parents than anyone else for fear of being kicked out of her home. "I feel like if there's anybody I'm more scared [to tell] I am bisexual it's my parents because ... if I ever were to bring it up I know I'd probably get kicked out of my house, or they just would disown me" (P8).

QPoC experience ostracism from both their racial communities and the LGBTQ+ community. In response to the question "do you feel that your sexual identity is stigmatized within your racial community?" all participants who answered gave an affirmative response. "Homophobia is in every community like the Black community, the Asian community, the white community ... They are only accepting of drag culture because they can get behind it. Drag queens are fun but when it comes to actual people that are queer, they turn their backs on them" (P3). Another participant said that when he is in a Black community, people look at him with disdain because he likes to wear crop tops, body glitter, and things people typically associate with women. He says, "We should all know by now Black people have been through a lot, so we should all sick together, but people in the community don't like me because I'm gay" (P10).

Another participant explained feeling the need to lessen their racial identity in queer settings. "In general there are some instances in my day to day life that I would have to act [with] more queer qualities, queer conversation topics. Sometimes when I go into queer spaces there's this need to lessen my race, things relating to my racial identity. It's kind of like code switching ... I don't really get a chance to express both of those depending on who I hang out with" (P12).

Online Experiences. QPoC often experience discrimination in online spaces. For example, on dating apps such as Tinder and Grindr, users' profiles often have racist announcements such as, "Whites only," "no Blacks," "no Asians," and "no Hispanics" in their biographies. QPoC often experienced fetishization on these apps as well, which, participants shared, felt dehumanizing. P9 shares, "Since I'm one of the few black guys on Grindr around this area, I'm basically just either being... targeted or sought out after by older white men... So they essentially just think I'm an exotic creature, something they can experiment with. I don't feel comfortable with this. I'm basically everyone's experiment." $\mathrm{QPoC}$ also experience discrimination when playing video games. Participants shared their experiences being called racial and sexual/gender slurs when trying to enjoy video games. P6 avoids multiplayer online games because he "know[s] those communities to be 14 year olds saying f****t and racial slurs and all that stuff."

Further, there is a lack of positive representation of QPoC characters in the media, including on Netflix and YouTube, instead QPoC roles are often stereotypical and pigeonholed. "It bothers me sometimes when the only black representation I see is like ... we're represented as a thug or like the best ones are when we're presented as second character to the protagonist and we're used for comic relief and I'm just like ... why is it always like this" (P9). Participants shared that they do not often see QPoC in shows and movies, and when they do they are rarely the main character. "I don't think there is enough representation, but there is also not a lot of drive for Asian queer people to be ahead of movements" (P3). There is frustration at the lack of 
QPoC on YouTube, streaming sites, and within videogames. "I just generally don't see good black LGBTQ representation in the media. I think the major films are just with white actors and with white men" (P9).

\subsection{RQ2: ICTs Used to Cope With Stigma}

In total, participants discussed 12 different information communication technologies that they used to cope with the stigma they experienced.

Social Media. Various social media platforms serve as tools for coping, such as Twitter, Snapchat, Reddit, Discord, Instagram, and GroupMe. Participants used chatting features on many of these social media platforms. By connecting with other people, participants have been able to learn more about their identities and how to cope with stigma, as well as simply talking about things that interest them. Support groups were created to discuss topics such as Queer representation in movies/tv shows, and Skype groups are used to connect with others. Participants used chat features, talking/texting friends, and online therapy. Blog/forum applications provide users a chance to post content and create dialogues with other users, asking and answering questions. Many participants expressed the ease with which they could access information about their identities from others with the same identities through Reddit, Tumblr, and Discord.

Video Games. Several participants shared that they connect with others who play video games through Discord and have maintained many friendships over multiple years. While gaming, participants utilize the chat to communicate with others. These features can be used to text friends for emotional support or general complaining. Participants did not specify the exact consoles, games, and platforms they were using.

Texting. Participants mentioned using texting broadly to connect with friends and people of the same identity. It was not specified which services were used for texting.

Streaming Media. Streaming platforms participants used to cope include Netflix and YouTube. Many individuals gravitate towards the shows and movies that provide representation of their identity. Participants watched TV shows by themselves or with their friends, using their phone, laptop, or iPad most often.

Participants used the aforementioned 12 ICTs primarily for seven different reasons. Table 2 serves as a guide for the following section. See Table 2 for theme descriptions.

Table 2. Theme Descriptions

Themes Description




\begin{tabular}{|c|c|}
\hline Seeking QPoC Media & $\begin{array}{l}\text { Actively looking for positive representation of their } \\
\text { identity (ex. On Netflix, individuals are looking for } \\
\text { non-stereotyped versions of themselves reflected in } \\
\text { storylines) }\end{array}$ \\
\hline $\begin{array}{l}\text { Distraction \& } \\
\text { Escapism }\end{array}$ & $\begin{array}{l}\text { Seeking to distract themselves or escape from negative } \\
\text { mental states, unpleasant realities, and unpleasant } \\
\text { emotions. }\end{array}$ \\
\hline $\begin{array}{l}\text { Communicating and } \\
\text { Connecting with } \\
\text { Others }\end{array}$ & $\begin{array}{l}\text { Connecting with their community through following other } \\
\text { QPoC on social media or engaging in group chats with } \\
\text { people of similar identities. This is an active engagement, } \\
\text { i.e replying to a tweet, posting in a chat, or creating larger } \\
\text { groups. }\end{array}$ \\
\hline $\begin{array}{l}\text { Seeking a } \\
\text { Community }\end{array}$ & $\begin{array}{l}\text { Actively seeking a permanent community of individuals } \\
\text { with similar identities. This refers to attempts to find a } \\
\text { community without actually interacting with individuals. }\end{array}$ \\
\hline $\begin{array}{l}\text { Seeking Emotional } \\
\text { Support }\end{array}$ & $\begin{array}{l}\text { Connecting with others to discuss relatable experiences } \\
\text { and exploring the emotions they feel due to those } \\
\text { experiences. }\end{array}$ \\
\hline Identity Exploration & $\begin{array}{l}\text { Learning about their identity, or exploring various } \\
\text { identities that might align with theirs, by obtaining } \\
\text { information from different sources. }\end{array}$ \\
\hline
\end{tabular}

Table 3. Themes of Coping \& ICT Usage

\begin{tabular}{|c|c|c|c|c|c|c|}
\hline & $\begin{array}{l}\text { Seeking } \\
\text { QPoC } \\
\text { Media }\end{array}$ & $\begin{array}{l}\text { Distraction } \\
\text { and } \\
\text { Escapism }\end{array}$ & $\begin{array}{c}\text { Communicating } \\
\text { and } \\
\text { Connecting } \\
\text { with Others }\end{array}$ & $\begin{array}{l}\text { Seeking a } \\
\text { community }\end{array}$ & $\begin{array}{l}\text { Seeking } \\
\text { Emotional } \\
\text { support }\end{array}$ & $\begin{array}{c}\text { Identity } \\
\text { Exploration }\end{array}$ \\
\hline Instagram & $\mathrm{x}$ & $\mathrm{x}$ & $\mathrm{x}$ & $\mathrm{x}$ & & $\mathrm{x}$ \\
\hline Twitter & $\mathrm{x}$ & $\mathrm{x}$ & $\mathrm{x}$ & $\mathrm{x}$ & $\mathrm{x}$ & $\mathrm{x}$ \\
\hline Discord & $\mathrm{x}$ & & $\mathrm{x}$ & $\mathrm{x}$ & & $\mathrm{x}$ \\
\hline Tinder & & & $\mathrm{x}$ & & & \\
\hline Grindr & & $\mathrm{x}$ & $\mathrm{x}$ & & & \\
\hline GroupMe & & & $\mathrm{x}$ & $\mathrm{x}$ & $\mathrm{x}$ & $\mathrm{x}$ \\
\hline Tumblr & $\mathrm{x}$ & $\mathrm{x}$ & & $\mathrm{x}$ & $\mathrm{x}$ & $\mathrm{x}$ \\
\hline Reddit & $\mathrm{x}$ & $\mathrm{x}$ & $\mathrm{x}$ & $\mathrm{x}$ & $\mathrm{x}$ & $\mathrm{x}$ \\
\hline
\end{tabular}




\begin{tabular}{lccccc} 
Netflix & $\mathrm{x}$ & $\mathrm{x}$ & & & $\mathrm{x}$ \\
YouTube & $\mathrm{x}$ & $\mathrm{x}$ & & & \\
Video & & $\mathrm{x}$ & & & \\
Games & & & $\mathrm{x}$ & & \\
Texting & & 8 & 6 & 5 & 7 \\
Total & 7 & 8 & 8 & & \\
\hline
\end{tabular}

Distraction and Escapism. Participants used Instagram, Twitter, Grindr, Tumblr, Reddit, Netflix, YouTube, and Video Games to distract themselves and escape from stigma in their daily lives. These distractions came in the form of comedy sometimes. One participant mentioned, "this is a coping mechanism I didn't really think about, but being on twitter and looking at memes" (P6). While this participant mentioned Twitter specifically, others used streaming sites (like Netflix) that allowed them to binge watch shows to escape their realities. Some use platforms such as YouTube to relax by listening to "meditative sounds like healing bowls, those sound stuff that help me calm down," (P1) and autonomous sensory meridian response, known as ASMR.

Communicating and Connecting with Others. Communicating and connecting with others in this context specifically refers to engaging in direct interaction with an individual or with smaller groups. Participants frequently used Instagram, Twitter, Discord, Tinder, Grindr, GroupMe, Reddit, and texting to connect with others. As these ICTs are all social, participants were able to communicate with individuals similar to themselves, which gave them a sense of support. One participant said that being able to text and FaceTime gives them a "safe environment in any situation;" if they are feeling emotional, they can "text or FaceTime for reassurance" (P6).

Seeking QPoC Media. Of the 12 ICTs participants discussed, seven ICTs were used to specifically engage with $\mathrm{QPoC}$ media. There is a distinction in this category, because this engagement excluded explicit and direct interaction with other individuals. Participants used Instagram. Twitter, Discord, Tumblr, Reddit, Netflix, and YouTube. One participant mentioned doing this to cope "because seeing characters in media with these identities makes me feel better about my identity like when I have doubts or feel anxious about my identity" (P12).

Identity Exploration. Participants used ICTs to explore their identity and its complexities. Using Instagram, Twitter, Discord, GroupMe, Tumblr, Reddit, and Netflix, participants were able to find information and learn more about QPoC identity. One participant shares, "sometimes I find myself on Instagram looking at, like, cute couples. ... That's what really does it for me because in my mind it's like 'oh my gosh this could be me'" (P8). One way participants explore their identity is through seeing themselves represented on screen.

Seeking a Community. Seeking a community in this context refers to actively searching for and becoming a part of a large, well-established community. Using 
Instagram, Twitter, Discord, Tumblr, Reddit, and GroupMe, several participants were able to find communities such as college organizations to join. Other participants, however, struggled to find a community despite actively searching. Exemplifying this struggle, P7 shares, "it's hard to find a group of people that are just like me, like Indian queer people. I don't know where I would go to find a group of friends that would identify that way. I don't know if it's because I don't try enough to go look for it or if it's just hard to find" (P7).

Emotional Support. Participants turn to Twitter, GroupMe, Reddit, Tumblr, and Texting for emotional support from friends and others. This includes discussing relatable experiences and emotions and forming emotional connections with others in their community. One participant used Reddit to find memes and engaged with others on this platform to discuss how they felt. They shared, "one [account] that I follow is 'gay_irl.' They'll post like, memes that, if you're gay, is relatable to you" (P10).

\section{Discussion}

\subsection{Experience of Stigma for QPoC}

Racial and sexual/gender prejudice pervade a vast range of settings. As a result, QPoC are constantly reminded of societal stigma towards their identities, which can be overwhelming. QPoC endure covert and overt attacks against their identities in offline settings, such as with family, and in online settings such as on social media. This demonstrates how inescapable intersectional stigma can be. In line with the Minority Stress theory, these negative experiences have been shown to lead to depression, anxiety and general psychological distress $[40,12,27]$. To ameliorate these effects, it can be important for QPoC to have online and offline spaces, which are free of judgment and are encouraging and supportive of both the racial and sexual orientation/gender aspects of their identities. These spaces can range from group chats to organizations and clubs.

QPoC are often faced with the difficult to navigate dilemma of wanting to be their true selves but also being told by people who are important to them that there is something inherently wrong with who they are. This lack of support and reinforcement of heteronormativity ${ }^{3}$ from valued individuals and peers can have damaging effects on one's mental health, such as chronic elevated stress [28]. In addition, in environments like these, it can be easy to internalize feelings of rejection, furthering negative impacts on mental health and self-esteem. This further emphasizes the importance of safe and positive environments in which QPoC are free to express and explore their identities. Additionally, support from loved ones and other individuals one considers important can go a long way towards ameliorating the negative effects of stigma.

Moreover, consistent with previous work, participants reported experiencing discrimination against the LGBTQ+ part of their identity and racism within the

\footnotetext{
${ }^{3}$ Of, relating to, or based on the attitude that heterosexuality is the only normal and natural expression of sexuality [21].
} 
LGBTQ+ community $[18,3,34]$. Participants experience ostracism from their own communities: ostracism from the LGBTQ+ community for being PoC, and ostracism from PoC communities for being LGBTQ+. This can leave them feeling alone and like there is no place for them. Queer communities in general have high rates of depression, anxiety, somatization, and overall psychological distress [3]. Racial minorities in general struggle with excessive amounts of psychological distress [23]. QPoC experience a combination of these effects, as they belong to both groups. On top of this, it is often difficult to relate to and find comfort within even their own identity groups because of this double stigma and ostracism. It is important that there are supportive, welcoming environments that accept QPoC for all parts of their identities to help combat these effects.

\subsection{The College Context in Relation to ICTs}

All participants in this study are enrolled in colleges/universities in the Northeastern region of the United States. Several participants mentioned experiencing the effects of stigma on their college campus. This experience of stigma is particularly concerning for students of this age, as they are preparing for entry into the workforce while simultaneously receiving higher education and exploring their identities; LGBTQ identity exploration usually occurs later in life through the coming out process as a teenager or early adulthood [15]. Racial and ethnic minorities are an already underrepresented group in higher education [16]; experiences of discrimination and prejudice can create hostile social environments and negatively impact mental health [27].

This age group is more comfortable using technology than any other age group before them [14]; As Gen Z is a prevalent group in virtual spaces, it's important to make sure they are safe online. The online spaces QPoC turn to in order to cope with stigma tend to be stigma-laden spaces themselves. These spaces need to be safe to be effective rather than reductive towards QPoC mental health. Without completely safe virtual spaces for QPoC, individuals like these participants have fewer reliable spaces to turn to. Participants turned to different socializing applications to find community, explore their identity, see representation, and more. Experiences of online stigma in the same place where these participants seek comfort and help is a problem that individuals in Information Science and Human-Computer Interaction fields can work to improve or even eradicate if they take the needs of this specific population into consideration when designing platforms.

\subsection{ICTs Used to Cope with Stigma}

Consistent with prior work in this area, participants in our study developed coping mechanisms to help mitigate negative emotional consequences of stigma and ICTs were one tool used for this purpose [7, 12]. The main ICTs participants mentioned they use to cope include Instagram, Twitter, Discord, Tinder, Grindr, GroupMe, Tumblr, Reddit, Netflix, YouTube, Video Games, and Texting. Much like Kuper et al. in 2013 and Widerhold in 2014, this study also found that QPoC often turn to online 
communities to explore their identities and to build connections with others who have similar identities as them [7, 18]. Other ICTs were used for distraction and escapism, looking for QPoC media, identity exploration, seeking a community, and finding emotional support. Despite turning to these ICTs to feel safe from stigma endured offline, QPoC experience the same discrimination and stigma in these online spaces. Previous research has addressed this issue of nuanced experiences of support and stigma for LGBTQ+ individuals: stigma experiences from offline physical spaces can and are replicated from to online settings $[13,38,15]$.

On social media, such as Twitter, Tumblr, and Reddit, participants stated that there is an abundance of popular hate accounts which are not monitored closely. These hate accounts foster homophobia, transphobia, and white supremacy. On a large social platform such as Twitter, it is easy for misinformation to spread broadly and quickly [7]. This applies to misinformation regarding QPoC as a community, resulting in the spread of stigma, misrepresentation, and misunderstanding of QPoC individuals.

Streaming services, such as Netflix and YouTube, overwhelmingly lack positive QPoC representation and depict a copious amount of heteronormativity. Many participants actively sought tv shows and movies and YouTubers that they felt they could connect with. Finding this positive representation is not as easy as one would expect due to a lack of representative casting, stereotypical roles, and an evident bias in the algorithms used to recommend shows and movies, which often exclude QPoC media. This creates poor representation of real people who identify as QPoC and invalidates queer identities by supporting heteronormative standards [26].

Using video games to escape from the real world becomes difficult when hate speech runs rampant in multi-player online games. The ease of being able to make hurtful, derogatory comments online is taken advantage of by many players, as they have no direct contact with one another and can remain anonymous behind a username or alias. On dating apps, such as Tinder and Grindr, participants often faced comments that directly fetishize their identities. Many QPoC turn to gaming and online dating to relax and to feel in control but are faced with the same stressors of the real world from which they are trying to escape.

Across the different modes of communication the participants used, the ability to communicate and connect with a community of individuals with the same or similar identities recurred most frequently. The sense of community amongst individuals who already feel alienated and discriminated against because of their identity offline is important as it offers a place of belonging and security. However, it should be acknowledged that these safe spaces QPoC turn to online for support and community also often harbor discrimiantion.

Due tof the duality of online communities, it is crucial that the positive experiences that QPoC have in these spaces are amplified. This includes connecting with others, sharing experiences, making/building friendships, and learning about identities from others. Simultaneously, there should be a stronger effort to monitor and remove individuals who foster negative experiences for others online. The ICTs participants reported using are not perfect, they contain many flaws as stigma permeates these spaces as well, however they can each be improved through the implementation of certain design suggestions which can make these spaces safer and more comfortable for this niche community overall. 


\subsection{Participant Suggestions for ICT Improvements}

Participants suggested improvements they felt would be largely accommodating to queer people of color to effectively cope with the consequences of stigma against their identities. These design implications span enhancements of the user interface of different social media apps, customization of algorithms within streaming sites and social media, creation of new apps, allocation of online resources by professionals, and censoring of hate speech/violence across social media platforms.

User Interface. Several participants suggested a desire for a QPoC-specific platform with an increase in easy to access group chats to connect with individuals with the same identities, as many struggle to find spaces as racial minorities within the LGBTQ+ community. A posting feature would also be ideal to post content for one another to learn from and enjoy. Group chats could also be created under the flag of a specific identity within the LGBTQ + community for users to join and communicate with others who identify with the same sexual orientation or gender easily. For example, anyone who identifies as asexual can join an asexual group chat. Participants connected similarities of this interface with Instagram or Snapchat, but specifically for the LGBTQ+ community.

One participant suggested the ability to search for specific memes they felt catered to their identity in a way that allows them to search text in pictures. Another participant expressed that very few games allow players to choose genders outside of the binary. They expressed a desire for the ability to simply choose masculine or feminine qualities and features instead of having to be boxed into a certain identity. This would aid in making queer relationships normalized and more safe within different games.

Algorithms. Participants suggested improvements to remedy the lack of QPoC representation in algorithms across streaming sites and social media. The problem with these algorithms, participants shared, is that as a whole they promote heteronormativity. In the gaming community, YouTube, Facebook, Twitter, streaming sites, participants felt as though they had to go out of their way to find queer content. It was suggested that a Black version of YouTube be created to share content with people of similar interests and experiences to stop conforming to white community standards. In addition to an increase in QPoC content suggestions on YouTube, there should be an increase in movies and tv shows on streaming platforms featuring QPoC.

One participant also suggested there be a better muting system on Twitter, as the current algorithm continues to show accounts and advertisements that users have blocked. This participant stated it isn't healthy to have constant reminders of individuals or ideas one has muted as it is very draining - especially if they are spreading misinformation and hate speech.

Dating Apps. The creation of apps that cater to one's sexual/gender identity would be ideal for QPoC. In most instances, apps such as Tinder, and Bumble cover a large 
range of individuals, however one participant suggested having an app or site exclusively for people who identify as asexual, for example, as they find it hard finding asexual people in their area on existing dating apps and websites.

Resources. Participants shared that when looking for identity-related, online resources from official organizations, such as their universities, they have only been able to find information from student organizations. Colleges and universities should allocate money and other resources towards finding more professionals to provide resources for the queer community.

Censoring. Across many social media, participants cited a flaw in the way many platforms handle accounts that promote hate speech and violent rhetoric. It is often ignored by those in charge of these platforms. Several participants believe Twitter and Facebook, specifically, as well as other platforms, need to increase censorship of white supremacists and shut down hate accounts and decrease the suspension of QPoC accounts for trivial reasons. There should also be more conversations about how white supremacists are enabled on these platforms and how they affect society as a whole.

\subsection{Authors' Design Suggestions}

Due to the overwhelming problems that exist in the ICTs QPoC currently use to cope, these design implications should be taken into account as an improvement of these platforms to be a safer, more inclusive environment for QPoC. For instance, we would recommend that for social media sites, such as Twitter, Tumblr, and Reddit, there could be more vigilance in shutting down discriminatory hate accounts. In the case of streaming services, such as YouTube and Netflix, there could be a better representation of $\mathrm{QPoC}$ and less heteronormative/more $\mathrm{QPoC}$ inclusive algorithms. For video games, there needs to be more effective monitoring and shutting down accounts of people who use slurs and other discriminatory language against other players. Finally, for dating apps, there needs to be more vigilant censoring and deleting of racist accounts as well as apps designed specifically for QPoC to connect/date others of similar identities.

The suggestions listed above could be taken into account when designing new apps or for innovation of said apps to promote inclusion and equality for all, no matter the race/ethnicity, sexual orientation, or gender.

\section{$6 \quad$ Limitations and Future Work}

There are three major limitations in this study that can be addressed in future research. First, the sample size limits the diversity represented as part of this study. To improve upon this work, there should be more participants of various racial identities included in future studies. Second, due to the focus on college-aged QPoC, the findings may 
not reflect what takes place for QPoC who are younger, older, or who are not part of an educational environment. Third, as the researchers had access to limited resources, only one participant from each institution was compensated (this was randomly determined). With an increase in funding, more participants would receive compensation, potentially increasing the number of overall participants. An improvement upon these limitations can help guide future iterations of the present research.

One suggestion for further research is to create a survey based off of the present research participants' experiences, coping strategies, and suggestions. The purpose of this survey would be to better understand how ICTs can be improved for QPoC coping. It is possible to expand this qualitative study to become a quantitative study to determine the extent to which the improvements put forth in this paper are desired by QPoC across a broad geographic range, thus increasing generalizability .

Another suggestion for future work is to use this information to engage in community participatory sessions. It would be helpful for companies, such as Twitter, and $\mathrm{QPoC}$ users to communicate directly with one another so the experts in the stigmatized experiences themselves could help create better platforms.

\section{Conclusion}

This study highlights the experience of double stigmatization queer people of color face and how they use ICTs to cope with the stigma of being part of two marginalized groups. Through semi-structured interviews, authors found that $\mathrm{QPoC}$ face this unique stigma both online and offline and that QPoC use many different types of ICTs to cope with this in various ways. QPoC use ICTs, such as Instagram, Twitter, Discord, Tinder, Grindr, GroupMe, Tumblr, Reddit, Netflix, YouTube, Video Games, and Texting. These digital tools are used to distract and escape, communicate and connect with others, seek a community, find emotional support, search for QPoC media, and to explore one's identity. Given the current state of society and the stigma they face, this niche community should be given the proper respect and support they deserve. Pervasive stigma has the potential to be addressed, and even lessened, by acting on the suggestions put forth in this paper. It is not on QPoC to do all of the work, it is on the world at large to progress. One way to further this progress is through improvements in HCI which can increase positive online experiences for QPoC and decrease the negative impact of stigma. Racial and sexual/gender stigma cannot be fixed overnight, but these findings can be used to improve upon existing ICTs in order to create a more inviting, safe, and inclusive space for Queer people of Color.

\section{Acknowledgements}

Thank you to the iSchool Inclusion Institute as well as to our mentors at our individual institutions for the guidance and support in conducting this study. 


\section{References}

1. Alegría, M., Pérez, D. J., \& Williams, S. (2003). The role of public policies in reducing mental health status disparities for people of color. Health Affairs, 22(5), 51-64. https://doi.org/10.1377/hlthaff.22.5.51

2. Balsam, K. F., Molina, Y., Beadnell, B., Simoni, J., \& Walters, K. (2011). Measuring multiple minority stress: The lgbt people of color microaggressions scale. Cultural Diversity and Ethnic Minority Psychology, 17(2), 163-174. https://doi.org/10.1037/a0023244

3. Braun, V., \& Clarke, V. (2006). Using thematic analysis in psychology. Qualitative research in psychology, 3(2), 77-101.

4. Castillo, C. "Information Credibility on Twitter." Proceedings of the 20th International Conference on World Wide Web, In: Association for Computing Machinery, pp. 675-684 (2011)

5. Cyrus, K. (2017). Multiple minorities as multiply marginalized: Applying the minority stress theory to LGBTQ people of color. Journal of Gay \& Lesbian Mental Health, 21(3), 194-202. http://dx.doi.org/10.1080/19359705.2017.1320739

6. Devito M., Walker A., Birnholtz J “Too Gay for Facebook”. Proceedings of the ACM on Human-Computer Interaction (2018)

7. Dimock M (2020) Defining generations: Where Millennials end and Generation Z begins. In: Pew Research Center.

https://www.pewresearch.org/fact-tank/2019/01/17/where-millennials-end-and-genera tion-z-begins/. Accessed 16 Oct 2020

8. Fry R, Parker K (2018) Early Benchmarks Show 'Post-Millennials' on Track to Be Most Diverse, Best-Educated Generation Yet. In: Pew Research Center's Social \& Demographic Trends Project. https://www.pewsocialtrends.org/2018/11/15/early-benchmarks-show-post-millennial s-on-track-to-be-most-diverse-best-educated-generation-yet/. Accessed 26 Sep 2020

9. Green ER, Peterson EN (2003)In: Glossary of LGBTQ-Related Terms. https://gbtq.ecu.edu/definitions/. Accessed 10 Oct 2020

10. Heteronormative. In: Merriam-Webster. https://www.merriam-webster.com/dictionary/heteronormative. Accessed 16 Oct 2020

11. Kuper, L. E., Coleman, B. R., \& Mustanski, B. S. (2013). Coping With LGBT and Racial-Ethnic-Related Stressors: A Mixed-Methods Study of LGBT Youth of Color. Journal of Research on Adolescence, 24(4), 703-719. doi: 10.1111/jora.12079

12. McInroy, Lauren B., and Shelley L. Craig. "Perspectives of LGBTQ Emerging Adults on the Depiction and Impact of LGBTQ Media Representation." Journal of Youth Studies, vol. 20, no. 1, Jan. 2017, pp. 32-46. Taylor and Francis $+N E J M$, doi:10.1080/13676261.2016.1184243.

13. Mink MD, Lindley LL, Weinstein AA (2014) Stress, Stigma, and Sexual Minority Status: The Intersectional Ecology Model of LGBTQ Health. Journal of Gay \& Lesbian Social Services 26:502-521. doi: 10.1080/10538720.2014.953660

14. Rho E., Haimson O., Andalibi N., Mazmanian M., Hayes, G. "Class Confessions: Restorative Properties in Online Experiences of Socioeconomic Stigma.” Proceedings of the 2017 CHI Conference on Human Factors in Computing Systems (2017)

15. Rivera, E., Poldruhi, M., Ward, C., Jenkins, G., Nichols, E., Pinter, A. "The black at the end of the rainbow: Online discrimination among LGBTQ African Americans" IConference 2019 Proceedings. Presented at the iConference 2019. (2019) 
16. Shield A., "Tend to Prefer Sane, Masculine, Caucasian (No Offense to Other Flavours Though)': Racial-Sexual Preferences, Entitlement, and Everyday Racism." Immigrants on Grindr: Race, Sexuality and Belonging Online In: Springer International Publishing (2019)

17. Smith A, Rainie L, Zickuhr K (2020) College students and technology. In: Pew Research Center: Internet, Science \& Tech. https://www.pewresearch.org/internet/2011/07/19/college-students-and-technology/. Accessed 16 Oct 2020

18. Smith, H.W., IPSOS Connect: Kids Are Alright - Understanding Gen Z, https://www.ipsos.com/sites/default/files/ct/publication/documents/2017-11/ipsos-mo ri-almanac-2017.pdf.

19. Walker A., Devito M. "'More gay' fits in better: Intracommunity Power Dynamics and Harms in Online LGBTQ+ Spaces.” Proceedings of the 2020 CHI Conference on Human Factors in Computing Systems (2020)

20. Whitfield, D. L., Walls, N. E., Langenderfer-Magruder, L., \& Clark, B. (2014). Queer Is the New Black? Not So Much: Racial Disparities in Anti-LGBTQ Discrimination. Journal of Gay \& Lesbian Social Services, 26(4), 426-440. doi: $10.1080 / 10538720.2014 .955556$

21. Wiederhold, B. K. (2014). Cyberbullying and lgbtq youth: A deadly combination. Cyberpsychology, Behavior, and Social Networking, 17(9), 569-570. https://doi.org/10.1089/cyber.2014.1521

22. (2018) Educational Attainment, by Race and Ethnicity - Race and Ethnicity in Higher Education. In: Race and Ethnicity in Higher Education. https://www.equityinhighered.org/indicators/u-s-population-trends-and-educational-at tainment/educational-attainment-by-race-and-ethnicity/. Accessed 20 Sep 2020

23. (2020) LGBTQ+ glossary for parents. In: Parent Info.

https://parentinfo.org/article/lgbtq-glossary-for-parents. Accessed 16 Oct 2020 